\title{
Pharmacological properties of baicalin on liver diseases: a narrative review
}

\author{
Jin-yu Yang ${ }^{1} \cdot$ Min $\mathrm{Li}^{1}$. Cheng-liang Zhang ${ }^{1}$. Dong Liu ${ }^{1}$ (])
}

Received: 29 September 2020 / Revised: 22 January 2021 / Accepted: 1 February 2021 / Published online: 17 February 2021

(c) The Author(s) 2021

\begin{abstract}
Baicalin is the main active component of Scutellaria baicalensis, widely used in traditional Chinese medicine thanks to its various pharmacological effects, such as anti-tumor, anti-inflammatory, and antibacterial properties, as well as cardiovascular, hepatic, and renal protective effect. Recently, the protective effects of baicalin on liver disease have received much more attention. Several studies showed that baicalin protects against several types of liver diseases including viral hepatitis, fatty liver disease, xenobiotic induced liver injury, cholestatic liver injury, and hepatocellular carcinoma, with a variety of pharmacological mechanisms. A comprehensive understanding of the mechanism of baicalin can provide a valuable reference for its clinical use, but up to now, no narrative review is available that summarizes the pharmacological effects of baicalin to clarify its potential use in the treatment of liver diseases. Therefore, this review summarizes the progress of baicalin research and the underlying mechanism in the treatment of various liver diseases, to promote further research and its clinical application.
\end{abstract}

Keywords Baicalin $\cdot$ Hepatitis $\cdot$ Fatty liver disease $\cdot$ Xenobiotic-induced liver injury $\cdot$ Hepatocellular carcinoma $\cdot$ Liver ischemia reperfusion

\section{Introduction}

Liver diseases are the main cause of illness and death worldwide [1,2]. Approximately $25 \%$ of adults in the world suffer from non-alcoholic fatty liver disease, and approximately 75 million are diagnosed with alcohol-related disorders and are at risk of alcohol-associated liver disease [3]. The global prevalence of viral hepatitis remains high, while drug-induced liver injury continues to increase as the main cause of acute hepatitis. Approximately 1.16 million deaths and 788,000 deaths occur each year due to cirrhosis and liver cancer, respectively [3,4]. Vaccination or newer drugs may reduce or relieve the burden of these liver diseases in developed countries, but the above remedies are still limited in developing countries [3]. Therefore, it is of utmost

Cheng-liang Zhang

clzhang@tjh.tjmu.edu.cn

Dong Liu

Liudong@tjh.tjmu.edu.cn

1 Department of Pharmacy, Tongji Hospital Affiliated Tongji Medical College, Huazhong University of Science and Technology, 1095 Jiefang avenue, Wuhan 430030, Hubei, China importance to find novel ways to effectively prevent the development and progress of liver diseases.

Baicalin possesses potential therapeutic effects on systemic diseases, including ocular disorders [5], periodontal diseases [6], inflammatory disorders [7], metabolic disorders [8] and even nervous system diseases [9, 10], thanks to its anti-inflammatory, anti-oxidative, anti-angiogenesis, and immunoregulatory effect. Moreover, baicalin possesses anti-obese, anti-viral, and anti-dyslipidemia effect, thus playing a critical role in improving liver function after injury or alleviating liver diseases [7, 11-13]. Since baicalin has a very wide range of pharmacological effects, more and more in-depth studies have been conducted. However, till now, no comprehensive review is available that summarizes the pharmacological effects of baicalin to clarify its potential use in the treatment of liver diseases. Therefore, the purpose of this review is to provide an update on the effects of baicalin and its mechanisms of action in the regulation of liver diseases. 


\section{Plant source, isolation, and properties of baicalin}

Baicalin $(\mathrm{C} 21 \mathrm{H} 18 \mathrm{O} 11$; 5,6,7-trihydroxyflavone-7- $\beta$-Dglucuronide; Fig. 1a) is the main chemical and pharmacological component of Scutellaria baicalensis (SB) [14]. SB is often been called Huangqin or skullcap, is the dried root of Scutellaria baicalensis Georgi, and has been extensively used in the treatment of several diseases, including hepatitis, hyperlipidemia, dysentery, and liver cirrhosis in China and neighboring countries $[15,16]$. Scutellaria baicalensis Georgi mainly grows in temperate regions and tropical mountains (with an altitude of approximately 1300-3000 m) in China, Russia's Easter Siberia, Mongolia, North Korea, and Japan [17]. It is harvested in spring and autumn to remove the fibrous roots and sediments, then dried in the sun after removing the rough skin.

As the main flavonoid component, baicalin in SB is present at a concentration of at least $9.0 \%$, making it as the quality standard of SB according to the Chinese Pharmacopoeia [18]. The conventional methods to purify baicalin from SB are solvent extraction and high-speed countercurrent chromatography [19]. Nowadays, the present method to separate baicalin is more efficient and advantageous, with high efficiency and less solvent waste due to the advanced technology used [20].
As a flavonoid bearing glycoside, baicalin shows low water solubility $(67.03 \pm 1.60 \mu \mathrm{g} / \mathrm{ml})$ and low permeability $\left(P_{\text {app }}=0.037 \times 10^{-6} \mathrm{~cm} / \mathrm{m}\right)[21]$, thus resulting in a low bioavailability after oral administration. The physicochemical properties of baicalin such as $\log \mathrm{P}=1.27, \mathrm{pKa} 1=7.6$, $\mathrm{pKa} 2=10.1$, were determined at $25^{\circ} \mathrm{C}$ in $0.1 \mathrm{M} \mathrm{NaCl}$ [22]. Ling et al. demonstrated that baicalin by itself is a poor radical scavenger (TEAC $=1.12, \mathrm{pH} 7.4$ ) and a poor antioxidant against free radical initiated lipid oxidation in liposomes, but this limitation can be reversed via the interaction with $\beta$-carotene [22]. In addition, $\gamma$-cyclodextrin complexation of baicalin can significantly increase its solubility by about 5 times. Thus, the physicochemical and structural properties of baicalin provide the data needed to design the dosage[23].

\section{The in vivo disposition of baicalin}

A mutual transformation of baicalin and its aglycone, baicalein (Fig. 1b) occurs in the body once baicalin is absorbed. Indeed, baicalin is hydrolyzed to baicalein by $\beta$-glucuronidase in the intestine soon after administration, and then, baicalein is transformed back to baicalin by UDPglucuronosyltransferase once it enters the systemic circulation [24]. Thus, baicalin is the major component in the systemic circulation rather than baicalein [25]. Baicalin concentration slightly increases in plasma (12-24 h) after oral administration, with a $\mathrm{T}_{1 / 2 \mathrm{TER}}$ of $12.1 \mathrm{~h}$ [26]. After i.v.
Fig. 1 Structure of baicalin and main metabolites
A<smiles>O=C(O)C1CC(Oc2cc3oc(-c4ccccc4)cc(=O)c3c(O)c2O)C(O)C2(O)OC1C2O</smiles>

baicalin

B<smiles>[R20]c1cc2oc(-c3ccccc3)cc(=O)c2c([R20])c1[R2]</smiles>

metabolites baicalein: $\mathrm{R}=\mathrm{H} ; \mathrm{R}^{\prime}=\mathrm{H} ; \mathrm{R}^{\prime \prime}=\mathrm{H}$

M8: R=GluA; R'=Glc; R"=H

M9: R=GluA; R'=GluA; R"=H

M17: R=Glc; R'=H; R"=H

M19: R=GluA; R'=CH3; R"=H

M25: R=GluA; R'=H; R"=H

M30: $\mathrm{R}=\mathrm{H} ; \mathrm{R}^{\prime}=\mathrm{CH} 3 ; \mathrm{R}^{\prime \prime}=\mathrm{H}$ 
administration, the elimination half-life $\left(\mathrm{T}_{1 / 2}\right)$ and the terminal elimination half-life $\left(\mathrm{T}_{1 / 2 \mathrm{TER}}\right)$ of baicalin were $0.1 \mathrm{~h}$ and $9.7 \mathrm{~h}$ in plasma, respectively [26]. In addition, the peak plasma concentration of baicalin $\left(\mathrm{C}_{\max }, 0.91 \pm 0.12 \mathrm{nmol} /\right.$ $\mathrm{ml}$ ) is reached at $5 \mathrm{~h}$ after oral administration. According to the area under the curve (AUC), the absolute bioavailability (F\%) of baicalin is $2.2 \pm 0.2 \%$, while one of its conjugated metabolites is $27.8+5.6 \%$ [26], indicating that bacalin is distributed in vivo mainly in the form of conjugates. The transport speed and distribution volume of baicalin depend on its binding degree with plasma proteins. The in vitro binding of baicalin to human serum proteins ranges from 86 to $92 \%$, suggesting that baicalin may affect the effective concentration of other co-administered drugs [27]. Moreover, baicalin can pass the blood-brain barrier, thus it is able to exert protective effects against various neurodegenerative disorders $[9,10]$.

UDP-glucuronosyltransferase and $\beta$-glucuronidase are crucial metabolic enzymes involved in the biotransformation of baicalin. Indeed, five biliary metabolites were identified in the rat bile after oral administration of baicalin. Among them, the main metabolites are 6-o- $\beta$-glucopyranuronosylbaicalein-7-o-sulfate and baicalein 6,7-di-o- $\beta$ glucupyranuroside [28]. These conjugated metabolites are then hydrolyzed to baicalein by $\beta$-glucuronidase/sulfatase in the gastrointestinal tract [28]. A total of 32 baicalin metabolites were identified by Zhang et al. in rat plasma, urine, and various tissues using more efficient approaches [29]. They found that baicalin in vivo undergoes several chemical transformations, such as methylation, hydrolysis, hydroxylation, methoxylation, glucuronide conjugation, sulfate conjugation, and their composite reactions. Five and 9 metabolites were found in rat liver and kidney, respectively, demonstrating that the rat liver and kidney are the most important organs for the distribution of baicalin after metabolism. In addition, six metabolites (M8, M9, M17, M19, M25, M30; Fig. 1c) may be related to the pharmacological effects of baicalin in vivo [29].

Baicalin is mainly excreted by the bile in the form of glucuronides and undergoes enterohepatic circulation [28]. Multidrug resistance-associated protein 2 is the main apical transporter of baicalin mediating the biliary efflux in the hepatocytes [30]. Indeed, the biliary excretion of baicalin is significantly decreased in multidrug resistance-associated protein 2 deficient rats, along with a markedly increased baicalin concentration in the plasma [31]. The fraction of baicalin and its conjugated metabolites is much less in the urine compared with the fraction in the bile [32]. The total urinary clearance of baicalein and baicalin is $<0.1 \%$, while approximately $27 \%$ of baicalein is excreted in the stools as an unchanged drug [25].

The pharmacokinetics of baicalin can help the understanding of its pharmacological effects in the liver. The enterohepatic circulation ensures that baicalin is particularly concentrated in the liver, which is beneficial in the treatment of liver diseases. However, its metabolites such as baicalein also play a role in the treatment of liver diseases [33, 34], suggesting that the underline mechanisms of baicalin against liver diseases may involve the effect of its metabolites. Since baicalin is mainly metabolized in the liver, co-administration of baicalin with other herbs/drugs may affect its in vivo properties, ultimately affecting its effects. Therefore, the therapeutic dosage of baicalin should be carefully explored not only because of the above factors but also due to its low bioavailability, and techniques to enhance its absorption or liver targeting should be developed.

\section{Effect of baicalin on liver diseases}

\section{Viruses hepatitis}

Viral hepatitis is an infectious disease mainly caused by a variety of hepatitis viruses and represents a major health problem threatening human health worldwide. More than 240 million people are infected with HBV every year, and more than 80,000 patients die from HBV-related liver disease $[35,36]$. Thus, it is of utmost importance to discover effective therapeutic drugs for its treatment.

Plant-derived flavonoids have certain advantages in combating viral infections, and their mechanisms of action include the prevention of virus invasion, inhibition of virus transcription, and its gene expression [37]. The flavonoid prescription containing baicalin exerts a significant curative effect in combating duck hepatitis A virus by the inhibition of its replication, the increase of $\mathrm{T}$ and $\mathrm{B}$ lymphocyte, the alleviation of liver damage, and the reduction of oxidative stress [38-40]. The combination of baicalin with catechin (both flavonoids) induces the cells to produce type I interferon $(\mathrm{IFN}-\alpha / \beta)$ that significantly impairs the stability of HBV RNA, capsid, and cccDNA or reduced the amount of HBV replicative intermediates, transcripts, and HBV surface antigen levels [41]. Baicalin alone also improves the viability of duck embryonic hepatocytes infected by duck hepatitis A virus, reducing the virus reproduction in these hepatocytes and inhibiting the transcription of HBV RNA by down-regulating HNF- $1 \alpha$ and HNF- $4 \alpha$ expression [42, 43].

The combined application of baicalin and entecavir has a better good synergistic effect, better than the effect they exert when they are used alone since the combination exerts an enhanced virus suppression, induces a natural immune response, and controls HBV-related liver inflammation [43]. The synergistic effect may be due to the different targets of the two drugs, thus overcoming the obstacle of the nucleoside drugs currently used that only block HBV replication but they are ineffective in HBV cccDNA accumulation and 
HBV surface antigen loss. These results suggest that baicalin may be useful in the combination therapy against viral hepatitis.

\section{Fatty liver diseases (FLD)}

The increased hepatic lipogenesis and serum non-esterified fatty acid lead to an excessive accumulation of lipids in the liver, causing fatty liver, impaired liver function, and eventually liver failure [44-46]. Baicalin is a potential therapeutic drug against FLD thanks to its several effects against it. Indeed, baicalin enhances lipid metabolism and represses hepatic de novo lipogenesis through the inhibition of the $\mathrm{Ca} 2+/ \mathrm{CaM}$-dependent protein kinase $\beta / \mathrm{AMP}$-activated protein kinase/acetyl-CoA carboxylase (CaMKK $\beta / A M P K /$ ACC) pathway [45, 47, 48]. In addition, baicalin directly binds carnitine palmitoyltransferase 1 , consequently promoting the lipid influx into mitochondria where they are oxidated [49], down-regulating lipogenesis genes such as sterol regulatory element-binding protein- $1 \mathrm{c}$, fatty acid synthase, and peroxisome proliferator-activated receptor alpha to reduce hepatic lipid accumulation $[46,47,50]$.

Another ability of baicalin is to suppress liver fibrosis, systemic inflammation, and oxidative stress in FLD. Indeed, it inhibits the expression of $\alpha$-smooth muscle actin, transforming growth factor beta 1 and collagen type I alpha 1 chain to reverse liver fibrosis [44-46]. Furthermore, it reverses the epithelial-mesenchymal transition through the inhibition of the TGF- $\beta 1 / \mathrm{Smad} 3$ pathway in vitro to prevent the development of liver fibrosis [51]. In addition, baicalin exerts an indirect antioxidant function by upregulating hepatic glutathione and superoxide dismutase levels and downregulating malondialdehyde levels in mice subjected to a high fat and high cholesterol diet [44]. Moreover, baicalin reduces systemic inflammation by blocking NLR pyrin domain containing 3-gasdermin D signaling, as demonstrated by in vitro experiments, and by the decreased release of pro-inflammatory factors via the inhibition of tolllike receptor 4 (TLR4) signaling cascade in mice [44, 46, 52-54].

Finally, baicalin alleviates glucose intolerance, hyperglycemia, and insulin resistance in mice under a diet inducing obesity. Baicalin significantly decreases insulin concentrations due to a high-fat diet, and the underlying mechanisms are related to the inhibitory effect of baicalin on the expression of p-p38, MAPK, p-CREB, FoxO1, PGC- $1 \alpha$, PEPCK and G6Pase [45, 47, 55]. Thus, baicalin may prevent FLD thanks to its multiple pharmacological effects.

\section{Xenobiotic-induced liver injury}

Baicalin exerts potential benefits on liver injury induced by some xenobiotics, such as drugs and environmental toxins.
It significantly attenuates oxidative stress and inflammatory response in acetaminophen (APAP)-treated mice [56-58]. Besides, baicalin stimulates the detoxification of APAP by preventing APAP-induced depletion of glutathione and suppresses CYP2E1 activity, the latter responsible for the production of $\mathrm{N}$-acetyl-p-benzoquinoneimine, a cytotoxic APAP intermediate metabolite [59].

LPS-induced liver injury is mainly related to excessive inflammation in the liver. Yanqiu et al. [60] found that baicalin decreases cell apoptosis and inflammatory reaction due to LPS through the upregulation of taurine upregulated gene 1 and the inactivation of both p38MAPK and Jun N-terminal kinase pathways, as well as through the reduction of NF- $\mathrm{KB}$ translocation. The underlying mechanism is associated to the up-regulation of the activity and expression of heme oxygenase 1 [61].

Iron overload is one of the most common causes of metalrelated liver toxicity. It leads to a significant increase in serum ALT and AST levels and SOD activity, and a decrease in the sulfhydryl content and glutathione peroxidase activity [62]. Baicalin not only alleviates hepatic damage due to iron accumulation, but also reverses the above abnormal serum indicators and the nitration level, and reduces serum iron content $[63,64]$. Baicalin also ameliorates cadmium chloride-induced hepatic cytotoxicity and confers protection against CCl4-induced liver injury through the reduction of the inflammatory response, hepatic fibrosis, and oxidative damage [65-68]. Similarly, baicalin ameliorates ethanolinduced liver injury through the modulation of hepatic oxidative stress, inflammation, and Sonic Hedgehog signaling pathway in rats $[69,70]$. Consequently, baicalin shows great therapeutic potential against various xenobiotics that induce liver injury, thus deserving further exploration.

\section{Hepatocellular carcinoma (HCC)}

HCC causes the death of more than 600,000 people each year and has become the third leading cause of cancerrelated death worldwide [71]. Less than $30 \%$ of patients with HCC have the opportunity to be subjected to surgery due to their poor physical condition, thus, the 5 year survival rate of these patients is only $9 \%[71,72]$.

Baicalin shows a potential therapeutic effect against HCC through the induction of cell apoptosis and autophagy in the tumor. Baicalin inhibits HCC cell viability and proliferation in vitro by down-regulating the expression of cyclin A, cyclin-dependent-kinase 2 and cyclin D1 and inducing apoptosis [73, 74], while it significantly inhibits the growth of xenografts in vivo in nude mice[73]. In addition, Baicalin induces cell apoptosis in HepG2 and SMMC-7721 cells through the up-regulation of Bax, the down-regulation of Bcl-2, the induction of the cleavage of Caspase- 9 and Caspase-3, and the induction of poly ADP-ribose polymerase 
[73]. The underlying mechanism may be potentially related to the ability of baicalin to activate the transcription factor 6 signaling pathway through targeting the site- 2 protease in HCC cells [74]. Furthermore, baicalin suppresses the growth of HCC by inducing cell autophagy via the regulation of tumor-associated macrophages repolarization [75]. Despite all these discoveries, the knowledge of the pharmacological mechanisms used by baicalin to regulate $\mathrm{HCC}$ inhibition is still limited, and further studies are needed to provide additional evidence for a potential application of baicalin as alternative medicine in $\mathrm{HCC}$ treatment.

\section{Liver ischemia reperfusion (IR) injury}

Liver IR occurs in several clinical circumstances, including liver transplantation, partial hepatic resection, shock, and hepatic failure [76, 77]. IR injury begins with the onset of hypoxia, which causes cellular damage, followed by the restoration of the blood flow and oxygen delivery, which exacerbates cellular damage $[78,79]$. At present, no effective prevention strategy is available against liver IR injury.

Baicalin attenuates oxidative damage, increases cell viability and decreases the level of lactate dehydrogenase [80], suggesting a potential protective effect on hepatic IR injury. Indeed, baicalin significantly increases HO-1 expression and reduces inducible nitric oxide synthase, cyclooxygenase-2, TNF receptor 1-associated protein expression, and toll-like receptor 4-mediated inflammatory response in liver IR animals [81, 82]. Moreover, baicalin inhibits the mitochondrial swelling rate and caspases- 3 and 8 activation in IR rats [82]. Furthermore, baicalin exerts a protective effect on liver IR injury through the induction of cell autophagy, but the detailed mechanisms regulating autophagy in liver IR injury should be further investigated by both in vivo and in vitro experiments [80].

\section{Cholestatic liver injury}

Cholestasis is a bile flow disorder caused by various factors, and it is characterized by an accumulation of toxic bile acids in the liver, causing the damage of hepatocytes and bile ducts and eventually liver fibrosis or liver failure [83]. Thus, an acceleration in the flow and excretion of bile acids and their reduced accumulation are the key points to consider in the development of a drug to combat cholestasis [84]. Co-administration of the Scutellaria baicalensis extract and metformin promotes the conversion of cholesterol to bile acids and accelerates their fecal excretion [85]. Shen et al. found that baicalin administration in mice significantly alleviates intrahepatic cholestasis and fibrosis after bile duct ligation [86]. Moreover, our previous work demonstrated that baicalin exerts a protective effect on estrogen-induced cholestatic liver injury via the induction of the sirtuin 1/ hepatic nuclear receptor-1a/FXR signaling pathway, consequently maintaining hepatic bile acid homeostasis and alleviating hepatic inflammation $[87,88]$. However, the protective effect of baicalin on cholestatic liver injury needs further investigation in the future due to the complex pathogenesis of this injury, and multi-omics technology might be used to improve our knowledge on this protective effect.

\section{Liver targeting strategy of baicalin}

Although baicalin exhibits many biological activities against liver disease, its low lipid and water solubility limits its clinical application. To be more effective against liver diseases, the drugs should specifically target the liver, thus, the clinical application of baicalin should also meet this aspect. This is why different baicalin-based formulations and drug delivery systems have been studied in order to improve the absorption and bioavailability of baicalin and enhance its effect on liver disease. For example, the baicalin-loaded liposome formulation improves the lipophilicity of baicalin and further enhances its concentration in the liver [89, 90]. Indeed, this formulation exerts a greater effect in the treatment of non-alcoholic fatty liver disease than baicalin alone [54]. In addition, Iman et al. [74, 91] used lactobionic acidmodified-chitosan lactate polymer to obtain a site-specific baicalin delivery to increase the liver targeting ability of this drug. As expected, the concentration of baicalin in the liver after oral administration was remarkably increased and the mean area under the curve $\left(\mathrm{AUC}_{0-24}\right)$ was much higher after the use of this polymer as a delivery system. These strategies show great potential in the increase of the bioavailability of baicalin in the liver after oral administration.

\section{Advantages and safety of baicalin in liver diseases}

Clinical management of liver diseases is still rather challenging due to late diagnosis, progressive characteristic, therapeutic drugs with a poor effect, and limited therapeutic strategies $[1,3]$. Several drugs are commonly used in the treatment of liver diseases, such as ursodeoxycholic acid [92], cholestyramine [93], corticosteroids [94], and simvastatin [95], but with barely satisfactory clinical results. However, an increasing number of active molecules and small molecule inhibitors have been found to be hepatoprotective [96-99]. Most of them are poorly studied and the underline mechanisms and toxic effects are still unclear. Baicalin is derived from SB, which has been extensively used throughout China's long history to combat liver diseases, and baicalin itself has also been applied in clinical practice for decades $[14,16]$. Distinguished from the above hepatic active 
molecules, baicalin presents diversified pharmacological action and hepatoprotective effects, such as anti-viral, antioxidant, anti-inflammatory and anti-obesity effects, resulting a potential candidate against liver diseases (Supplementary Table 1; Fig. 2.).

A study showed that baicalin may have potential nephrotoxic effects in rats at high doses $(800-1600 \mathrm{mg} / \mathrm{kg}$ ), although no toxic to the liver of rats [100]. At the administered dose, no evident toxic and side effects of baicalin or its aglycone baicalein were observed in many clinical studies. A study showed that a single oral dose of $100-2800 \mathrm{mg}$ baicalein is safe and well-tolerated by healthy subjects, with no signs of toxicity in the liver or kidney [25]. Besides, the capsules of baicalin have been applied in clinical practice as an adjuvant treatment of acute and chronic hepatitis and evasive hepatitis. Zhang et al. found no abnormal behavior in ICR mice at the maximum dose of capsules containing $15 \mathrm{~g} /$ $\mathrm{kg}$ baicalin after oral administration. Moreover, no death occurred among the mice within one week of administration, and no abnormalities were observed in any organ after dissection. These results demonstrated that baicalin capsules possess a good safety profile [101]. Thus, the favorable safety profile of baicalin merits further studies for its clinical use. Overall, baicalin may be a promising therapeutic option in the treatment of liver diseases.

\section{Conclusion}

Liver diseases represent a significant burden of disease and death worldwide. The development of liver disease is related to a variety of factors such as alcohol, drugs, viruses, and obesity, and the mechanisms involved are several, including inflammation, oxidative stress, liver fibrosis, and apoptosis. Currently available drugs are not ideal for treating liver diseases, thus, new drugs are still needed. SB has been widely used for thousands of years in the treatment of liver and gallbladder diseases. Baicalin is the main chemical component and the primary pharmacologically active compound of SB. This article summarizes the wide range of pharmacological properties of baicalin, such as antioxidant, antiviral, antiinflammatory, anti-obesity, and anti-tumor effects, thus it

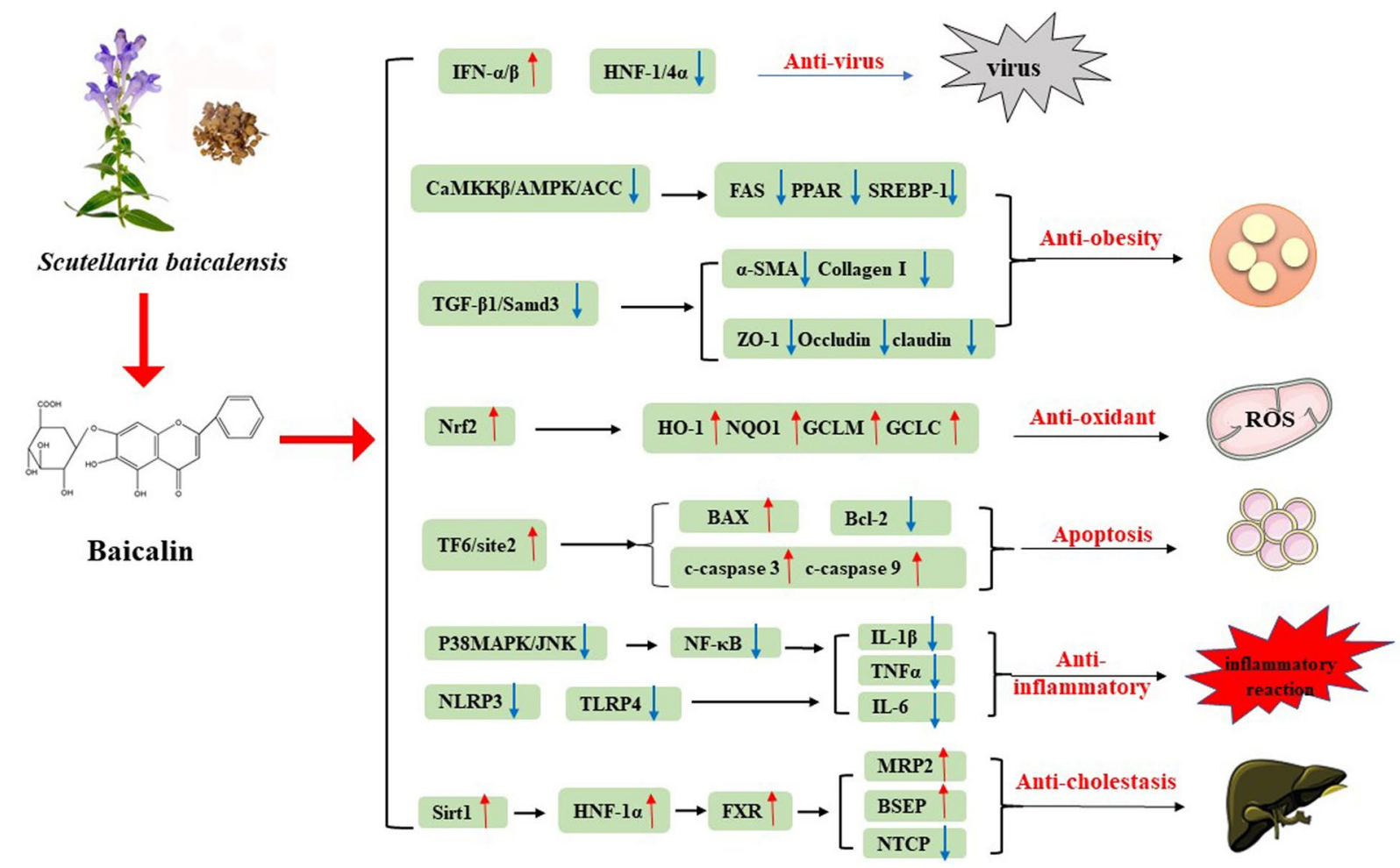

Fig. 2 Main pharmacological mechanisms of baicalin on liver diseases: (I) Baicalin induces the production of IFN- $\alpha / \beta$ and down-regulates the expression of HNF-1/4 $\alpha$ to reduce the replication of HBV; (II) Baicalin inhibits the CaMKK $\beta /$ AMPK/ACC and TGF- $\beta 1 / \mathrm{Smad} 3$ signaling pathway, thus represses hepatic de novo lipogenesis and prevents the development of liver fibrosis; (III) baicalin activates the transcription factor 6 signaling pathway through targeting the site- 2 protease to induce cell apoptosis in HCC cells; IV, baicalin increases the Nrf2/HO-1 signaling pathway to attenuate oxidative damage; $\mathrm{V}$, baicalin decreases the release of pro-inflammatory factors by blocking NLRP3 or TLRP4 or p38MAPK/JNK/NF- $\mathrm{BB}$ pathways and improves systemic inflammation; (VI) baicalin maintains hepatic bile acid homeostasis via the induction of the sirt1/HNF-1 $\alpha /$ FXR signaling pathway. The red arrows indicate the pathways which are up-regulated, and the blue arrows mean the pathways that are downregulated by baicalin 
might be of great value in combating liver diseases (Supplementary Table 1; Fig. 2). A comprehensive understanding of the mechanism of action of baicalin might provide a valuable basis and reference for its clinical use. Nevertheless, extensive studies are still needed to discover its key targets, and clarify its clinical indications. Indeed, the knowledge of baicalin mechanism of action is still limited to animals and in vitro studies, and it is still necessary to further explore the underlying mechanisms to verify its effects and safety in clinical trials. Besides, the low hydrophilic property and poor absorption of baicalin limit its application. Thus, new technologies and new preparations are urgently needed and should be developed to improve its solubility and liver targeting ability. Although baicalin is potentially effective in the management of several liver diseases described in this review, its role in autoimmune liver disease is scarcely reported. Therefore, more basic and applied studies should be performed to comprehensively clarify the pharmacological mechanisms and application of baicalin in the treatment of liver diseases.

Supplementary Information The online version contains supplementary material available at https://doi.org/10.1007/s43440-021-00227-1.

Acknowledgement This work was supported by the National Natural Science Foundation of China (NO.82073939).

Author contributions $\mathrm{J}-\mathrm{yY}$ wrote this manuscript. ML performed the search. C-1Z and DL were responsible for the idea and modified this manuscript. All authors reviewed and approved the manuscript.

\section{Compliance with ethical standards}

Conflict of interest The authors declared no potential conflicts of interest with respect to the research, authorship, and/or publication of this article.

Open Access This article is licensed under a Creative Commons Attribution 4.0 International License, which permits use, sharing, adaptation, distribution and reproduction in any medium or format, as long as you give appropriate credit to the original author(s) and the source, provide a link to the Creative Commons licence, and indicate if changes were made. The images or other third party material in this article are included in the article's Creative Commons licence, unless indicated otherwise in a credit line to the material. If material is not included in the article's Creative Commons licence and your intended use is not permitted by statutory regulation or exceeds the permitted use, you will need to obtain permission directly from the copyright holder. To view a copy of this licence, visit http://creativecommons.org/licenses/by/4.0/.

\section{References}

1. Peter B. The global burden of liver disease: a challenge for methods and for public health. BMC Med. 2014;12:159. https://doi. org/10.1186/s12916-014-0159-5.
2. Jia X, Fei W, Nai-Kei W, Jinhan H, Rui Z, Ruijuan S, et al. Global liver disease burdens and research trends: analysis from a Chinese perspective. J Hepatol. 2019;71(1):212-21. https://doi. org/10.1016/j.jhep.2019.03.004.

3. Sumeet KA, Harshad D, John E, Patrick SK. Burden of liver diseases in the world. J Hepatol. 2019;70(1):151-71. https://doi. org/10.1016/j.jhep.2018.09.014.

4. Mokdad AA, Lopez AD, Shahraz S, Lozano R, Mokdad AH, Stanaway J, Murray CJ, Naghavi M. Liver cirrhosis mortality in 187 countries between 1980 and 2010: a systematic analysis. BMC Med. 2014;12:145. https://doi.org/10.1186/s1291 6-014-0145-y.

5. Xiao JR, Do CW, To CH. Potential therapeutic effects of baicalein, baicalin, and wogonin in ocular disorders. J Ocul Pharmacol Th. 2014;30(8):605-14. https://doi.org/10.1089/jop.2014.0074.

6. Jiang M, Li ZN, Zhu GX. Protective role of flavonoid baicalin from Scutellaria baicalensis in periodontal disease pathogenesis: a literature review. Complement Ther Med. 2018;38:11-8. https ://doi.org/10.1016/j.ctim.2018.03.010.

7. Dinda B, Dinda S, DasSharma S, Banik R, Chakraborty A, Dinda $M$. Therapeutic potentials of baicalin and its aglycone, baicalein against inflammatory disorders. Eur J Med Chem. 2017;131:6880. https://doi.org/10.1016/j.ejmech.2017.03.004.

8. Fang PH, Yu M, Shi MY, Bo P, Gu XW, Zhang ZW. Baicalin and its aglycone: a novel approach for treatment of metabolic disorders. Pharmacol Rep. 2020;72(1):13-23. https://doi.org/10.1007/ s43440-019-00024-x.

9. Sowndhararajan K, Deepa P, Kim M, Park S, Kim S. Neuroprotective and cognitive enhancement potentials of baicalin: a review. Brain Sciences. 2018;8(6):104. https://doi.org/10.3390/ brainsci8060104.

10. Liang W, Huang XB, Chen WQ. The effects of baicalin and baicalein on cerebral ischemia: a review. Aging Dis. 2017;8(6):850 67. https://doi.org/10.14336/AD.2017.0829.

11. Kuo YT, Lin CC, Kuo HT, Hung JH, Liu CH, Jassey A, et al. Identification of baicalin from Bofutsushosan and Daisaikoto as a potent inducer of glucose uptake and modulator of insulin signaling-associated pathways. J Food Drug Anal. 2019;27(1):240-8. https://doi.org/10.1016/j.jfda.2018.07.002.

12. Yang MD, Chiang YM, Higashiyama R, Asahina K, Mann $\mathrm{D}$, Mann J, et al. Rosmarinic acid and baicalin epigenetically de-repress Ppary in hepatic stellate cells for their anti-fibrotic effect. Hepatology. 2012;55(4):1271-81. https://doi.org/10.1002/ hep. 24792 .

13. Yonghong W, Yanru J, Xin Y, Bin L, Hongjuan G, Taotao Y. A potential role of Baicalin to inhibit apoptosis and protect against acute liver and kidney injury in rat preeclampsia model. Biomed Pharmacother. 2018;108:1546-52. https://doi.org/10.1016/j. biopha.2018.09.107.

14. Bochořáková H, Paulová H, Slanina J, Musil P, Táborská E. Main flavonoids in the root of Scutellaria baicalensis cultivated in Europe and their comparative antiradical properties. Phytother Res. 2003;17(6):640-4. https://doi.org/10.1002/ptr.1216.

15. Zhao Q, Chen X, Martin C. Scutellaria baicalensis, the golden herb from the garden of Chinese medicinal plants. Sci Bull (Beijing). 2016;61(18):1391-8. https://doi.org/10.1007/s1143 4-016-1136-5.

16. Song J, Long J, Xie L, Zhang L, Xie Q, Chen H, et al. Applications, phytochemistry, pharmacological effects, pharmacokinetics, toxicity of Scutellaria baicalensis Georgi. and its probably potential therapeutic effects on COVID-19: a review. Chin Med. 2020;15:102. https://doi.org/10.1186/s13020-020-00384-0.

17. Shang X, He X, He X, Li M, Zhang R, Fan P, et al. The genus Scutellaria an ethnopharmacological and phytochemical review. J Ethnopharmacol. 2010;128(2):279-313. https://doi. org/10.1016/j.jep.2010.01.006. 
18. Commission CP. Pharmacopoeia of the People's Republic of China. Beijing: People's Medical Publishing House; 2005.

19. Wu S, Sun A, Liu R. Separation and purification of baicalin and wogonoside from the Chinese medicinal plant Scutellaria baicalensis Georgi by high-speed counter-current chromatography. J Chromatogr A. 2005;1066(1-2):243-7. https://doi.org/10.1016/j. chroma.2005.01.054.

20. Du Z, Wang K, Tao Y, Chen L, Qiu F. Purification of baicalin and wogonoside from Scutellaria baicalensis extracts by macroporous resin adsorption chromatography. J Chromatogr B Analyt Technol Biomed Life Sci. 2012;908:143-9. https://doi.org/10.1016/j. jchromb.2012.09.024.

21. Wu H, Long X, Yuan F, Chen L, Pan S, Liu Y, et al. Combined use of phospholipid complexes and self-emulsifying microemulsions for improving the oral absorption of a BCS class IV compound, baicalin. Acta Pharm Sin B. 2014;4(3):217-26. https:// doi.org/10.1016/j.apsb.2014.03.002.

22. Liang R, Han R, Fu L, Ai X, Zhang J, Skibsted LH. Baicalin in radical scavenging and its synergistic effect with $\beta$-Carotene in antilipoxidation. J Agric Food Chem. 2009;57(15):7118-24. https://doi.org/10.1021/jf9013263].

23. Jakab G, Bogdán D, Mazák K, Deme R, Mucsi Z, Mándity IM, et al. Physicochemical profiling of baicalin along with the development and characterization of cyclodextrin inclusion complexes. AAPS PharmSciTech. 2019;20(8):314. https://doi. org/10.1208/s12249-019-1525-6.

24. Noh K, Kang Y, Nepal M, Jeong K, Oh D, Kang M, et al. Role of intestinal microbiota in baicalin-induced drug interaction and its pharmacokinetics. Molecules. 2016;21(3):337. https://doi. org/10.3390/molecules21030337.

25. Li M, Shi A, Pang HX, Xue W, Li Y, Cao GY, et al. Safety, tolerability, and pharmacokinetics of a single ascending dose of baicalein chewable tablets in healthy subjects. J Ethnopharmacol. 2014;156:210-5. https://doi.org/10.1016/j.jep.2014.08.031.

26. Xing J, Chen XY, Zhong DF. Absorption and enterohepatic circulation of baicalin in rats. Life Sci. 2005;78(2):140-6. https:// doi.org/10.1016/j.lfs.2005.04.072.

27. Tang Y, Zhu HY, Zhang YY, Huang CG. Determination of human plasma protein binding of baicalin by ultrafiltration and high-performance liquid chromatography. Biomed Chromatogr. 2006;20(10):1116-9. https://doi.org/10.1002/bmc.655.

28. Abe K, Inoue O, Yumioka E. Biliary excretion of metabolites of baicalin and baicalein in rats. Chem Pharm Bull. 1990;38(1):209-11 ([PMID:2337942]).

29. Zhang JY, Cai W, Zhou Y, Liu Y, Wu XD, Li Y, et al. Profiling and identification of the metabolites of baicalin and study on their tissue distribution in rats by ultra-high-performance liquid chromatography with linear ion trap-Orbitrap mass spectrometer. J Chromatogr B Analyt Technol Biomed Life Sci. 2015;985:91102. https://doi.org/10.1016/j.jchromb.2015.01.018.

30. Kalapos-Kovács B, Magda B, Jani M, Fekete Z, Szabó PT, Antal I, et al. Multiple ABC transporters efflux baicalin. Phytother Res. 2015;29(12):1987-90. https://doi.org/10.1002/ptr.5477.

31. Akao T, Sato K, Hanada M. Hepatic Contribution to a marked increase in the plasma concentration of baicalin after oral administration of its aglycone, baicalein, in multidrug resistance-associated protein 2-deficient rat. Biol Pharm Bull. 2009;32(12):2079-82. https://doi.org/10.1248/bpb.32.2079.

32. Lai MY, Hsiu SL, Chen CC, Hou YC, Chao PD. Urinary pharmacokinetics of baicalein, wogonin and their glycosides after oral administration of scutellariae radix in humans. Biol Pharm Bull. 2003;26(1):79-83. https://doi.org/10.1248/bpb.26.79.

33. Lai CC, Huang PH, Yang AH, Chiang SC, Tang CY, Tseng $\mathrm{KW}$, et al. baicalein reduces liver injury induced by myocardial ischemia and reperfusion. Am J Chin Med. 2016;44(3):531-50. https://doi.org/10.1142/S0192415X16500294.
34. Dong YS, Xing Y, Sun J, Sun WL, Xu YB, Quan CS. Baicalein alleviates liver oxidative stress and apoptosis induced by highlevel glucose through the activation of the PERK/Nrf2 signaling pathway. Molecules. 2020;25(3):599. https://doi.org/10.3390/ molecules25030599.

35. Ropero Álvarez AM, Pérez-Vilar S, Pacis-Tirso C, Contreras $\mathrm{M}$, El Omeiri N, Ruiz-Matus C, et al. Progress in vaccination towards hepatitis $\mathrm{B}$ control and elimination in the region of the Americas. Bmc Publ Health. 2017;17(1):325. https://doi. org/10.1186/s12889-017-4227-6.

36. Meireles LC, Marinho RT, Damme PV. Three decades of hepatitis B control with vaccination. World J Hepatol. 2015;7(18):2127-32. https://doi.org/10.4254/wjh.v7.i18.2127.

37. Tsukuda S, Watashi K, Hojima T, Isogawa M, Iwamoto M, Omagari K, et al. A new class of hepatitis B and D virus entry inhibitors, proanthocyanidin and its analogs, that directly act on the viral large surface proteins. Hepatology. 2017;65(4):1104-16. https://doi.org/10.1002/hep.28952.

38. Chen Y, Yuan WJ, Yang YH, Yao FK, Ming K, Liu JG. Inhibition mechanisms of baicalin and its phospholipid complex against DHAV-1 replication. Poultry Sci. 2018;97(11):3816-25. https ://doi.org/10.3382/ps/pey255.

39. Chen Y, Zeng L, Yang JJ, Wang YX, Yao FK, Wu Y, et al. Anti-DHAV-1 reproduction and immuno-regulatory effects of a flavonoid prescription on duck virus hepatitis. Pharm Biol. 2016;55(1):1545-52. https://doi.org/10.1080/13880 209.2017.1309554.

40. Chen Y, Zeng L, Lu Y, Yang YL, Xu MY, Wang YX, et al. Treatment effect of a flavonoid prescription on duck virus hepatitis by its hepatoprotective and antioxidative ability. Pharm Biol. 2017;55(1):198-205. https://doi.org/10.1080/13880 209.2016.1255977.

41. Pollicino T, Musolino C, Irrera N, Bitto A, Lombardo D, Timmoneri M, et al. Flavocoxid exerts a potent antiviral effect against hepatitis B virus. Inflamm Res. 2018;67(1):89-103. https://doi. org/10.1007/s00011-017-1099-2.

42. Chen Y, Yao FK, Ming K, Shi JT, Zeng L, Wang DY, et al. Assessment of the effect of baicalin on duck virus hepatitis. Curr Mol Med. 2019;19(5):376-86. https://doi.org/10.2174/15665 24019666190405095301.

43. Huang H, Zhou W, Zhu H, Zhou P, Shi X. Baicalin benefits the anti-HBV therapy via inhibiting HBV viral RNAs. Toxicol Appl Pharmacol. 2017;323:36-43. https://doi.org/10.1016/j. taap.2017.03.016.

44. Zhong X, Liu H. Baicalin attenuates diet induced nonalcoholic steatohepatitis by inhibiting inflammation and oxidative stress via suppressing JNK signaling pathways. Biomed Pharmacother. 2018;98:111-7. https://doi.org/10.1016/j.biopha.2017.12.026.

45. Xi YL, Wu MZ, Li HX, Dong SQ, Luo E, Gu MB, et al. Baicalin attenuates high fat diet-induced obesity and liver dysfunction: dose-response and potential role of CaMKK $\beta / \mathrm{AMPK} / \mathrm{ACC}$ Pathway. Cell Physiol Biochem. 2015;35(6):2349-59. https://doi. org/10.1159/000374037.

46. Zhang JL, Zhang HM, Deng XL, Zhang N, Liu BB, Xin SL, et al. Baicalin attenuates non-alcoholic steatohepatitis by suppressing key regulators of lipid metabolism, inflammation and fibrosis in mice. Life Sci. 2018;192:46-54. https://doi.org/10.1016/j. lfs.2017.11.027.

47. Guo HX, Liu DH, Ma Y, Liu JF, Wang Y, Du ZY, et al. Longterm baicalin administration ameliorates metabolic disorders and hepatic steatosis in rats given a high-fat diet. Acta Pharmacol Sin. 2009;30(11):1505-12. https://doi.org/10.1038/aps.2009.150.

48. Ma Y, Yang FZ, Wang Y, Du ZY, Liu DH, Guo HX, et al. $\mathrm{CaMKKb}$ is involved in AMP-activated protein kinase activation by baicalin in LKB1 deficient cell lines. PLoS ONE. 
2012;7(10):e47900. https://doi.org/10.1371/journal.pone.00479 00 .

49. Dai J, Liang K, Zhao S, Jia W, Liu Y, Wu H, et al. Chemoproteomics reveals baicalin activates hepatic CPT1 to ameliorate diet-induced obesity and hepatic steatosis. Proc Natl Acad Sci USA. 2018;115(26):E5896-905. https://doi.org/10.1073/ pnas.1801745115.

50. Chen Q, Liu MY, Yu HY, Li J, Wang S, Zhang Y, et al. Scutellaria baicalensis regulates FFA metabolism to ameliorate NAFLD through the AMPK-mediated SREBP signaling pathway. J Nat Med-tokyo. 2018;72(3):655-66. https://doi.org/10.1007/ s11418-018-1199-5.

51. Wu T, Liu T, Xing LJ, Ji G. Baicalin and puerarin reverse epithelial-mesenchymal transition via the TGF- $\beta 1 / \mathrm{Smad} 3$ pathway in vitro. Exp Ther Med. 2018;16(3):1968-74. https://doi. org/10.3892/etm.2018.6400.

52. Zhang J, Zhang H, Deng XL, Zhang YP, Xu KS. Baicalin protects AML-12 cells from lipotoxicity via the suppression of ER stress and TXNIP/NLRP3 inflammasome activation. Chem Biol Interact. 2017;278:189-96. https://doi.org/10.1016/j. cbi.2017.10.010.

53. Shi HL, Zhang YL, Xing J, Liu L, Qiao F, Li J, et al. Baicalin attenuates hepatic injury in non-alcoholic steatohepatitis cell model by suppressing inflammasome-dependent GSDMD-mediated cell pyroptosis. Int Immunopharmacol. 2020;81:106195. https://doi.org/10.1016/j.intimp.2020.106195.

54. Liu J, Yuan YY, Gong X, Zhang LK, Zhou Q, Wu SW, et al. Baicalin and its nanoliposomes ameliorates nonalcoholic fatty liver disease via suppression of TLR4 signaling cascade in mice. Int Immunopharmacol. 2020;80:106208. https://doi.org/10.1016/j. intimp.2020.106208.

55. Fang PH, Sun YB, Gu XR, Shi MY, Bo P, Zhang ZW, et al. Baicalin ameliorates hepatic insulin resistance and gluconeogenic activity through inhibition of $\mathrm{p} 38$ MAPK/PGC- $1 \alpha$ pathway. Phytomedicine. 2019;64:153074. https://doi.org/10.1016/j.phyme d.2019.153074.

56. Liao CC, Day YJ, Lee HC, Liou JC, Chou AH, Liu FC. Baicalin attenuates IL-17-mediated acetaminophen-induced liver injury in a mouse model. PLoS ONE. 2016;11(11):e166856. https://doi. org/10.1371/journal.pone.0166856.

57. Liao CC, Day YJ, Lee HC, Liou JT, Chou AH, Liu FC. ERK signaling pathway plays a key role in baicalin protection against acetaminophen-induced liver injury. Am J Chin Med. 2017;45(01):105-21. https://doi.org/10.1142/S0192415X175000 82.

58. Idiosyncratic NK, Hepatotoxicity D. Idiosyncratic Drug Hepatotoxicity. Nat Rev Drug Discov. 2005;4(6):489-99. https://doi. org/10.1038/nrd1750.

59. Jang SI, Kim HJ, Hwang KM, Jekal SJ, Pae HO, Choi BM, et al. hepatoprotective effect of baicalin, a major flavone fromscutellaria radix, on acetaminophen-induced liver injury in mice. Immunopharm Immunot. 2003;25(4):585-94. https://doi. org/10.1081/iph-120026443.

60. Huang YQ, Sun MY, Yang XF, Ma A, Ma YJ, Zhao AY. Baicalin relieves inflammation stimulated by lipopolysaccharide via upregulating TUG1 in liver cells. J Physiol Biochem. 2019;75(4):463-73. https://doi.org/10.1007/s13105-019-00698 -0 .

61. Wan JY, Gong X, Zhang L, Li HZ, Zhou YF, Zhou QX. Protective effect of baicalin against Lipopolysaccharide/d-galactosamine-induced liver injury in mice by up-regulation of Heme oxygenase-1. Eur J Pharmacol. 2008;587(1-3):302-8. https:// doi.org/10.1016/j.ejphar.2008.02.081.

62. Qiao HX, Han HC, Hong DS, Ren ZH, Chen Y, Zhou CX. Protective effects of baicalin on carbon tetrachloride induced liver injury by activating PPAR $\gamma$ and inhibiting TGF $\beta 1$.
Pharm Biol. 2011;49(1):38-45. https://doi.org/10.3109/13880 209.2010.493179.

63. Zhang Y, Huang Y, Deng XR, Xu Y, Gao ZH, Li HL. Iron overload-induced rat liver injury: Involvement of protein tyrosine nitration and the effect of baicalin. Eur J Pharmacol. 2012;680(13):95-101. https://doi.org/10.1016/j.ejphar.2012.01.010.

64. Zhao YL, Li HL, Gao ZH, Xu HB. Effects of dietary baicalin supplementation on iron overload-induced mouse liver oxidative injury. Eur J Pharmacol. 2005;509(2-3):195-200. https:// doi.org/10.1016/j.ejphar.2004.11.060.

65. Wen YF, Zhao JQ, Bhadauria M, Nirala SK. Baicalin prevents cadmium induced hepatic cytotoxicity, oxidative stress and histomorphometric alterations. Exp Toxicol Pathol. 2013;65(12):189-96. https://doi.org/10.1016/j.etp.2011.08.005.

66. Zhang LJ, Yu JP, Li D, Huang YH, Chen ZX, Wang XZ. Effects of cytokines on carbon tetrachloride-induced hepatic fibrogenesis in rats. World J Gastroenterol. 2004;10(1):77-81. https://doi. org/10.3748/wjg.v10.i1.77.

67. Peng XD, Dai LL, Huang CQ, He CM, Chen LJ. Correlation between anti-fibrotic effect of baicalin and serum cytokines in rat hepatic fibrosis. World J Gastroentero. 2009;15(37):4720-5. https://doi.org/10.3748/wjg.15.4720.

68. Park SW, Lee CH, Kim YS, Kang SS, Jeon SJ, Son KH, et al. Protective effect of baicalin against carbon tetrachloride-induced acute hepatic injury in mice. J Pharmacol Sci. 2008;106(1):13643. https://doi.org/10.1254/jphs.fp0071392.

69. Wang HF, Zhang YL, Bai RX, Wang M, Du SY. Baicalin attenuates alcoholic liver injury through modulation of hepatic oxidative stress. Cell Physiol Biochem. 2016;39(3):1129-40. https:// doi.org/10.1159/000447820.

70. Wang XX, Chang XH, Zhan HB, Zhang Q, Li CY, Yang MM, et al. Curcumin and Baicalin ameliorate ethanol-induced liver oxidative damage via the Nrf2/HO-1 pathway. J Food Biochem. 2020. https://doi.org/10.1111/jfbc.13425.

71. Ferenci P, Fried M, Labrecque D, Bruix J, Sherman M, Omata $\mathrm{M}$, et al. Hepatocellular carcinoma (HCC): a global perspective. J Clin Gastroenterol. 2010;44(4):239-45. https://doi.org/10.1097/ MCG.0b013e3181d46ef2.

72. Song PP, Tobe RG, Inagaki Y, Kokudo N, Hasegawa K, Sugawara $\mathrm{Y}$, et al. The management of hepatocellular carcinoma around the world: a comparison of guidelines from 2001 to 2011. Liver Int. 2012;32(7):1053-63. https://doi.org/10.111 1/j.1478-3231.2012.02792.x.

73. Yu Y, Pei MY, Li L. Baicalin induces apoptosis in hepatic cancer cells in vitro and suppresses tumor growth in vivo. Int J Clin Exp Med. 2015;8(6):8958-67.

74. Yu Z, Luo X, Wang C, Ye JH, Liu SR, Xie L, et al. Baicalin promoted site-2 protease and not site-1 protease in endoplasmic reticulum stress-induced apoptosis of human hepatocellular carcinoma cells. Febs Open Bio. 2016;6(11):1093-101. https://doi. org/10.1002/2211-5463.12130.

75. Zhang XJ, Tang X, Liu HQ, Li LX, Hou Q, Gao JM. Autophagy induced by baicalin involves downregulation of CD147 in SMMC-7721 cells in vitro. Oncol Rep. 2012;27(4):1128-34. https://doi.org/10.3892/or.2011.1599.

76. John RK, Tsung A. Molecular biology of liver ischemia/reperfusion injury: established mechanisms and recent advancements. Surg Clin North Am. 2010;90(4):665-77. https://doi. org/10.1016/j.suc.2010.04.003.

77. Nakamura K, Kageyama S, Kupiec-Weglinski JW. The evolving role of neutrophils in liver transplant ischemia-reperfusion injury. Curr Transplant Rep. 2019;6(1):78-89. https://doi.org/10.1007/ s40472-019-0230-4.

78. Carchman EH, Rao J, Loughran PA, Rosengart MR, Zuckerbraun BS. Heme oxygenase-1-mediated autophagy protects against hepatocyte cell death and hepatic injury from infection/sepsis in 
mice. Hepatology. 2011;53(6):2053-62. https://doi.org/10.1002/ hep. 24324 .

79. Wang Y, Shen J, Xiong XX, Xu YH, Zhang H, Huang CJ, et al. Remote ischemic preconditioning protects against liver ischemiareperfusion injury via heme oxygenase-1- induced autophagy. PLoS ONE. 2014;9(6):e98834. https://doi.org/10.1371/journ al.pone.0098834.

80. Liu F, Zhang J, Qian JM, Wu G, Ma ZY. Baicalin attenuates liver hypoxia/reoxygenation injury by inducing autophagy. Exp Ther Med. 2018;16(2):657-64. https://doi.org/10.3892/ etm.2018.6284.

81. Kim SJ, Moon YJ, Lee SM. Protective effects of baicalin against ischemia/reperfusion injury in rat liver. J Nat Prod. 2010;73(12):2003-8. https://doi.org/10.1021/np100389z.

82. Kim SJ, Lee SM. Effect of baicalin on toll-like receptor 4-mediated ischemia/reperfusion inflammatory responses in alcoholic fatty liver condition. Toxicol Appl Pharmacol. 2012;258(1):4350. https://doi.org/10.1016/j.taap.2011.10.005.

83. Gossard AA, Talwalkar JA. Cholestatic liver disease. Med Clin North Am. 2014;98(1):73-85. https://doi.org/10.1016/j. mcna.2013.09.002.

84. Xiang D, Yang JY, Liu YN, He WX, Zhang S, Li XP, et al. Calculus Bovis Sativus improves bile acid homeostasis via farnesoid $\mathrm{X}$ receptor-mediated signaling in rats with estrogeninduced cholestasis. Front Pharmacol. 2019;10:48. https://doi. org/10.3389/fphar.2019.00048.

85. Han K, Bose S, Wang JH, Lim SK, Chin YW, Kim YM, et al. In vivo therapeutic effect of combination treatment with metformin and Scutellaria baicalensis on maintaining bile acid homeostasis. PLoS ONE. 2017;12(9):e182467. https://doi. org/10.1371/journal.pone.0182467.

86. Shen KZ, Feng XW, Pan H, Zhang F, Xie HY, Zheng SS. Baicalin ameliorates experimental liver cholestasis in mice by modulation of oxidative stress, inflammation, and NRF2 transcription factor. Oxid Med Cell Longev. 2017;2017:1-11. https://doi. org/10.1155/2017/6169128.

87. Yang JY, Xiang DC, Xiang D, He WX, Liu YN, Lan LL, et al. Baicalin protects against $17 \alpha$-ethinylestradiol-induced cholestasis via the sirtuin $1 /$ hepatic nuclear receptor- $1 \alpha /$ farnesoid $X$ receptor pathway. Front Pharmacol. 2020;10:1685. https://doi. org/10.3389/fphar.2019.01685.

88. Zhang CL, Xu YJ, Xiang D, Yang JY, Lei K, Liu D. Pharmacokinetic characteristics of baicalin in rats with $17 \alpha$-ethynylestradial-induced intrahepatic cholestasis. Curr Med Sci. 2018;38(1):167-73. https://doi.org/10.1007/s11596-018-1861-x.

89. Li N, Feng LL, Tan YJ, Xiang Y, Zhang RQ, Yang M. Preparation, Characterization, pharmacokinetics and biodistribution of baicalin-loaded liposome on cerebral ischemia-reperfusion after i.v. administration in rats. Molecules. 2018;23(7):1747. https:// doi.org/10.3390/molecules23071747.

90. Wei YM, Guo JM, Zheng XL, Wu J, Zhou Y, Yu Y, et al. Preparation, pharmacokinetics and biodistribution of baicalin-loaded liposomes. Int J Nanomedicine. 2014;9:3623-30. https://doi. org/10.2147/IJN.S66312.

91. Ahmed IS, Rashed HM, Fayez H, Farouk F, Shamma RN. Nanoparticle-mediated dual targeting: an approach for enhanced baicalin delivery to the liver. Pharmaceutics. 2020;12(2):107. https ://doi.org/10.3390/pharmaceutics12020107.

92. Floreani A, Mangini C. Primary biliary cholangitis: old and novel therapy. Eur J Intern Med. 2018;47:1-5. https://doi. org/10.1016/j.ejim.2017.06.020.

93. Düll MM, Kremer AE. Treatment of pruritus secondary to liver disease. urr Gastroenterol Rep. 2019;21(9):48. https://doi. org/10.1007/s11894-019-0713-6.

94. Singh S, Osna NA, Kharbanda KK. Treatment options for alcoholic and non-alcoholic fatty liver disease: a review. World J Gastroenterol. 2017;23(36):6549-70. https://doi.org/10.3748/ wjg.v23.i36.6549.

95. Eslami L, Merat S, Malekzadeh R, Nasseri-Moghaddam S, Ara$\min \mathrm{H}$. Statins for non-alcoholic fatty liver disease and non-alcoholic steatohepatitis. Cochrane Database Syst Rev. 2013. https:// doi.org/10.1002/14651858.CD008623.pub2.

96. Gao L, Yang X, Li Y, Wang Z, Wang S, Tan S, et al. Curcumol inhibits KLF5-dependent angiogenesis by blocking the ROS/ ERK signaling in liver sinusoidal endothelial cells. Life Sci. 2020. https://doi.org/10.1016/j.lfs.2020.118696.

97. Han X, Ding C, Zhang G, Pan R, Liu Y, Huang N, et al. Liraglutide ameliorates obesity-related nonalcoholic fatty liver disease by regulating Sestrin2-mediated Nrf2/HO-1 pathway. Biochem Biophys Res Commun. 2020;525(4):895-901. https://doi. org/10.1016/j.bbrc.2020.03.032.

98. Engelmann C, Sheikh M, Sharma S, Kondo T, Loeffler-Wirth $\mathrm{H}$, Zheng YB, et al. Toll-like receptor 4 is a therapeutic target for prevention and treatment of liver failure. J Hepatol. 2020;73(1):102-12. https://doi.org/10.1016/j.jhep.2020.01.011.

99. Zhao CQ, Zhou Y, Ping J, Xu LM. Traditional Chinese medicine for treatment of liver diseases: progress, challenges and opportunities. J Integr Med. 2014;12(5):401-8. https://doi.org/10.1016/ S2095-4964(14)60039-X.

100. Cai Y, Ma W, Xiao Y, Wu B, Li X, Liu F, et al. High doses of baicalin induces kidney injury and fibrosis through regulating TGF- $\beta /$ Smad signaling pathway. Toxicol Appl Pharmacol 2017;333:1-9. https://doi.org/10.1016/j.taap.2017.08.003.

101. Zhang XP, Cheng QH, Zhang Y. Acute toxicity test of baicalin capsule in mice. J Med Res. 2006;09:16-9.

Publisher's Note Springer Nature remains neutral with regard to jurisdictional claims in published maps and institutional affiliations. 